\title{
ROLE OF OMEGA-3 FATTY ACID IN DRY EYE SYNDROME ASSOCIATED WITH MEIBOMITIS
}

\author{
Garima Rai1 ${ }^{1}$ Deepak Mishra², Ketki Khandhadiya ${ }^{3}$, Shailesh Kumar4
}

${ }^{1}$ Assistant Professor, Department of Ophthalmology, Heritage Institute of Medical Sciences, Varanasi. ${ }^{2}$ Assistant Professor, Department of Ophthalmology, Heritage Institute of Medical Sciences, Varanasi. ${ }_{3}^{3}$ Assistant Professor, Department of Microbiology, Heritage Institute of Medical Sciences, Varanasi. ${ }^{4}$ Assistant Professor, Department of Forensic Medicine, Heritage Institute of Medical Sciences, Varanasi.

\section{ABSTRACT}

\section{AIM}

To evaluate the role of dietary supplementation of Omega-3 fatty acid in treatment of dry eye associated with meibomitis.

\section{MATERIAL AND METHOD}

A prospective, interventional study was done at HIMS, Varanasi. Hundred eyes of fifty patients were studied for changes in dry eye status after starting $500 \mathrm{mg}$ of Omega-3 fatty acid [Eicosapentaenoic Acid $325 \mathrm{mg}$ and Docosahexaenoic Acid $175 \mathrm{mg}$ ] twice a day for three months. There were four patient visit (at baseline, 1 month, 2 month, and 3 month). On each visit recording of corrected distance visual acuity, slit lamp examination, questionnaire base symptom evaluation and scoring was done. Objective evaluation was done by Schirmer 1 test, Tear Film Break-Up Time (TBUT).

\section{RESULT}

A total of fifty patients participated in the study of which 35 patients were female and 15 were male. All the patients were in the age group 45 years-75 years mean being $60.0 \pm 5.5$ years. At last visit, $60 \%$ of symptomatic patients were asymptomatic and $40 \%$ had moderate improvement in symptom score. $>90 \%$ patient showed significant improvement in Schirmer test values by 3-month follow-up. On evaluating TBUT, nearly $80 \%$ patients had scores within normal range by the end of the study.

\section{CONCLUSION}

Recent advances in understanding the pathophysiology of DES has led to evolution of newer modalities of treatment. Omega-3 fatty acid modulate the inflammatory process in the body may have a promising role to play in dry eye.

\section{KEYWORDS}

Omega-3 Fatty Acid, Dry Eye, Meibomitis.

HOW TO CITE THIS ARTICLE: Rai G, Mishra D, Khandhadiya K, et al. Role of omega- 3 fatty acid in dry eye syndrome associated with meibomitis. J. Evolution Med. Dent. Sci. 2016;5(67):4790-4792, DOI: 10.14260/jemds/2016/1091

\section{INTRODUCTION}

Dry eye is produced due to tear film abnormality, stemming from aqueous deficiencies, or evaporation of tear film, 1 although it may also arise from lid closure abnormality or environmental condition. Current treatment are centered on the management of the tear film and temporary relief maybe achieved through instillation of artificial tears, which does not last long as underlying cause is still unattended. ${ }^{2}$ Up to $25 \%$ of patients consulting eye care practitioners present with dry eye symptoms. ${ }^{3}$ Dry eye has been defined by National Eye Institute as a disorder of the tear film due to tear deficiency or excessive tear evaporation, which causes damage to the interpalpebral ocular surface and is associated with symptoms of ocular discomfort. ${ }^{4}$ Dry eye can be divided into two definitive types: evaporative and aqueous deficient. ${ }^{5}$ There are many factors that can lead to Dry Eye Syndrome (DES) and both evaporative and aqueous deficient dry eye condition will result in increased tear osmolarity. ${ }^{6}$ Evaporative dry eye may arise due to a meibomian oil deficiency or lid inadequacies such as an

Financial or Other, Competing Interest: None.

Submission 19-05-2016, Peer Review 03-08-2016,

Acceptance 10-08-2016, Published 20-08-2016.

Corresponding Author:

Dr. Garima Rai,

\#B-201, Virat MS, Plot No. 166-167,

Manduadih, Varanasi, Uttar Pradesh.

E-mail: rai1garima@rediffmail.com

DOI: $10.14260 /$ jemds/2016/1091 incomplete blink or a low blink rate. 5 A deficiency of vitamin A, which is needed for maintaining health of epithelial tissues may also result in evaporative dry eye..$^{5}$ Aqueous deficient DES can be further broken down to Sjogren and non-Sjogren dry eye. In the non-Sjogren version, it may arise from a lacrimal duct obstruction ${ }^{5}$ resulting in increased tear osmolarity. ${ }^{7}$

Research has shown that dry eye and nutrition are not mutually exclusive, ${ }^{8}$ promising interest in the use of nutritional supplementation or dietary modification for the prevention and treatment of this condition. Essential Fatty Acid (EFA) may enhance the lipid layer of the tear film, thus retarding evaporation ${ }^{9}$ and improving dry eye. Eicosapentaenoic Acid (EPA) and Docosahexaenoic Acid (DHA) and alpha-Linolenic acid are the three omega-3 fatty acid that cannot be synthesised in the body and have to be supplemented in diet. EPA and DHA modulate prostaglandin metabolism towards anti-inflammatory prostaglandin synthesis due to competitive inhibition of arachidonic acid pathway. ${ }^{10}$ Inflammation also plays a significant role in DES. Increased concentration of cytokines such as interleukin 1, interleukin 6, and tumour necrosis factor alpha have been found in the tear film of dry eye patient. ${ }^{11}$

The present study was conducted to assess the effect of dietary supplementation of EFA in improving symptoms and clinical parameters of disease activity in patients with DES. 


\section{MATERIAL AND METHOD}

A prospective interventional, non-randomised study was conducted at Heritage Institute of Medical Sciences, Varanasi, a tertiary care centre. The institutional review board and local ethics committee approved the study. A written informed consent was obtained from all patients based on Helsinki protocol. The eligibility criteria for patient entering the study were previous clinical diagnosis of dry eye associated with meibomitis. Exclusion criteria were patient with previous ocular surgery, alteration of lacrimal drainage system, or eyelid abnormalities. Patients with any systemic disorder that might affect the ocular surface or pregnant and lactating mother were excluded as well. Patients using ophthalmic or systemic medication affecting tear secretion or lipid metabolism or treatment with commercially available Essential Fatty Acid (EFA) or vitamin supplement were excluded.

Primary outcome measures ( 3 months after intervention) were the change in subjective symptoms of dry eye. A score of 0-3 was assigned to the common symptom of dry eye such as itching or burning, sandy or gritty sensation, redness, blurring of vision, ocular fatigue, or excessive blinking. When absent-0, sometimes present-1, frequently present-2, and always present-3 (Score of 0-6 was mild, 6.1-12 was moderate and 12.1-18 was severe dry eye).

Secondary outcome measures (3 months after intervention) were the Schirmer's 1 test for tear production, Tear Film Break-up Time (TBUT), and Lissamine green.

Subjects were seen at baseline, 1 month and 3 month followup after beginning the supplementation. At each visit, detailed ocular examination consisting of BCVA and slit lamp evaluation including assessment of lid margin, eye lashes, and meibomian gland orifice for any blockage or stenosis. Platelet count, prothrombin time, and Activated Partial Thromboplastin Time (APTT) were also measured at each visit.

To avoid the effect of lid manipulation, TBUT was performed first and average of two successive readings was taken. After half an hour interval, Schirmer's test was performed in which readings were taken after anaesthetising the eye ball and keeping the lids closed for 5 minute. Thirty minutes after this Lissamine green test was performed, a questionnaire of dry eye symptom was provided at each visit and scores assigned for each symptom was assessed and total score calculated.

\section{RESULT}

A total of 50 patients participated in the study of which 35 patients were female and 15 were male falling in age group 45 75 years mean age being $60.00 \pm 5.5$ years.

\begin{tabular}{|c|c|c|c|}
\hline No. of Patients & Baseline & 1st Month & 3rd Month \\
\hline Mild & 10 & 20 & 15 \\
\hline Moderate & 30 & 10 & 5 \\
\hline Severe & 10 & 10 & 0 \\
\hline $\begin{array}{r}\text { Table 1: Changes in Ocular Surface Disease Index Score } \\
\text { after Omega-3 Fatty Acid Supplementation }\end{array}$
\end{tabular}

\begin{tabular}{|c|c|c|c|}
\hline & Baseline & 1st Month & 3rd Month \\
\hline$<5$ Seconds & 40 & 30 & 5 \\
\hline 5-10 Seconds & 10 & 15 & 5 \\
\hline$>10$ Seconds & 0 & 5 & 40 \\
\hline \multicolumn{2}{|c|}{ Table 2: Changes }
\end{tabular}

\begin{tabular}{|c|c|c|c|}
\hline & Baseline & 1 $^{\text {st }}$ Month & 3rd Month \\
\hline$<3 \mathrm{~mm}$ & 10 & 5 & 0 \\
\hline $4-7 \mathrm{~mm}$ & 30 & 10 & 5 \\
\hline $7-10 \mathrm{~mm}$ & 10 & 10 & 15 \\
\hline$>10 \mathrm{~mm}$ & nil & 15 & 30 \\
\hline \multicolumn{2}{|c|}{ Table 3: Schirmer Test Values Post-Intervention } \\
\hline
\end{tabular}

At last visit, $60 \%$ of the symptomatic patients were asymptomatic and $40 \%$ had moderate improvement in symptom score.

At the end of study none of the patients had Schirmer test $<3 \mathrm{~mm}$ and more than 30 patients had Schirmer test with in normal range. $>90 \%$ subjects showed significant improvement in Schirmer test values by 3-month follow up.

On evaluating TBUT, nearly $80 \%$ patients had scores with in normal range by the end of study. Nearly, all the patients showed improvement in TBUT values after EFA supplementation for three months.

\section{DISCUSSION}

Rosenberg and Asbell12 had analysed all literature published till date on correlation between Omega-3 fatty acid and dry eye and concluded that although correlation exist between fatty acid supplementation and dry eye disorder no strong conclusion can be made because of limitation in reported scientific studies.

To establish the association between Omega-3 fatty acid supplement and improvement in symptom and clinical parameter in DES associated with meibomian gland disease, we conducted this prospective intervention non-randomised study in Northern India where diets are devoid of Omega-3 fatty acid in contrast to Southern part and eastern part where fish is an essential component of diet.

There was a slight preponderance of female patients in our study. This could be explained by the fact that acne rosacea, keratoconjunctivitis sicca, and meibomian gland disease are more common in female probably due to different levels of androgen and dietary supplementation. ${ }^{13}$

At 3-month post-intervention nearly all the patients showed improvement in symptom score. Creuzot and colleagues also observed improvement of dry eye relative to symptoms in a placebo-controlled randomised trial in a small sample size of subjects. ${ }^{13}$

On evaluating TBUT score, nearly $80 \%$ patients showed improvement at the end of 3 months. This result was similar to a randomised trial by Macsai and colleagues in which TBUT as well as meibum score changed significantly from baseline at one year. ${ }^{14}$

It is likely that dietary supplement of Omega-3 fatty acid alters the composition of meibomian gland secretion and meibum quality in patients with meibomian gland disease and chronic blepharitis. However, we did not attempt to study meibum characteristics in the present study.

A significant improvement in Schirmer test score was seen in nearly all the patients by the end of the study. Similar findings were documented by Wojtowicz JC et al in their pilot study on dry eye patients. 15

\section{CONCLUSION}

Recent advances in understanding the pathophysiology of DES has led to evolution of newer modalities of treatment. Omega3 fatty acid modulate the inflammatory process in the body 
and nutritional supplementation may have a promising role to play in dry eye. Dietary intervention with Omega-3 fatty acid not only cause symptomatic improvement, but better clinical markers of dry eye.

\section{REFERENCES}

1. Bron AJ. Diagnosis of dry eye. Surv Ophthalmol 2001;45(Suppl 2):S221-6.

2. Calonge M. The treatment of dry eye. Surv Ophthalmol 2001;45(Suppl 2):S227-39.

3. Moss SE, Klein R, Klein BE. Prevalence of and risk factor for dry eye syndrome. Arch Ophthalmol 2000;118(9): 1264-8.

4. Lemp MA. Report of the national eye institute/industry workshop on clinical trials in dry eye. Contact Lens Association of Ophthalmologist Journal 1995;21(4): 221-32.

5. Foulks GN, Lemp MA, Jester JV, et al. Report of international dry eye workshop (DWES). The Ocular Surface 2007;5(2):65-203.

6. Gilbard JP. Dry eye and role of nutrition. Optometry Today 2004;4:34-41.

7. Gilbard JP, Rossi SR, Gray KL. A new rabbit model for keratoconjunctivitis sicca. Invest Ophthalmol Vis Sci 1987;28(2):225-8.
8. Caffrey BE. Influence of diet on tear function. Optom Vis Sci 1991;68(1):58-72.

9. Srinivasan S. Chee-Chew Y. Is there a role of nutritional supplements in dry eye? Annals Academy of Medicine, Singapore 2007;36(Suppl):S45-9.

10. Pinna A, Piccinnini P, Carta F. Effect of oral linoleic and gamma linolenic acid on meibomian gland dysfunction. Cornea 2007;26(3):260-4.

11. Amparo F, Dastjerdi MH, Okanobo A, et al. Topical interleukin 1 receptor antagonist for treatment of dry eye disease: a randomised clinical trial. JAMA Ophthalmol 2013;131(6):715-23.

12. Rosenberg ES, Asbell PA. Essential fatty acid in the treatment of dry eye. Ocular Surface 2010;8(1):18-28.

13. Creuzot C, Passimard M, Viau S, et al. Improvement of dry eye symptoms with polyunsaturated fatty acids. J Fr Ophthalmol 2006;29(8):868-73.

14. Macsai MS. The role of omega-3 dietary supplementation in blepharitis and meibomian gland dysfunction. (an AOS thesis). Trans Am Ophthalmol Society 2008;106:336-56.

15. Wojtowicz JC, Butovich I, Uchiyama E, et al. Pilot, prospective, randomised, double masked, placebocontrolled clinical trial of an omega-3 supplement for dry eye. Cornea 2011;30(3):308-14. 